\title{
DADOS DE EQUÍLIBRIO-LÍQUIDO-LÍQUIDO PARA O APRIMORAMENTO DO PROCESSO DE PURIFICAÇÃO DO BIODIESEL DE ALGODÃO.
}

\author{
A. M. M. BESSA ${ }^{1}$; R. S. PINHEIRO ${ }^{1}$; N. S. EVANGELISTA ${ }^{1}$; H. B. DE SANT'ANA ${ }^{1}$ E R. \\ S. DE SANTIAGO-AGUIAR ${ }^{1}$
}
${ }^{1}$ Universidade Federal do Ceará, Departamento do Engenharia Química E-mail para contato: alinembessa@gmail.com

\begin{abstract}
RESUMO - O estudo do equilíbrio líquido-líquido (ELL) é uma importante ferramenta na avaliação do processo de purificação de biodiesel. Dados de ELL foram determinados para o sistema contendo biodiesel de algodão, água e metanol a $40{ }^{\circ} \mathrm{C}$, por intermédio do uso do método da densimetria. A confiabilidade dos dados foi avaliada com a equação de Othmer-Tobias, que apresentou coeficiente de regressão próximo a 1. Baixos valores do coeficiente de distribuição indicam maior afinidade do metanol pela fase rica em água e altos valores de seletividade apontam a água como um bom extrator. Foi realizada modelagem termodinâmica utilizando os seguintes modelos de contribuição de grupos: UNIFAC, UNIFACLLE e UNIFAC-Dortmund, apresentando desvios médios quadráticos globais iguais a 3,52\%, 4,22\% e 4,73\%, respectivamente. Estes valores de desvios indicam uma boa representação dos dados experimentais, uma vez que foram levados em consideração a composição global dos ésteres presentes no biodiesel.
\end{abstract}

\section{INTRODUÇÃO}

O interesse na diversificação da matriz energética mundial cresceu bastante nos últimos anos, devido principalmente a diminuição dos recursos de origem fóssil, ao aumento da demanda de energia e os problemas ambientais causados pela emissão de gases poluentes para a atmosfera. O biodiesel surgiu como um combustível alternativo em substituição ao óleo diesel derivado de petróleo, por apresentar características ambientalmente favoráveis quando comparado como diesel convencional. Além disso, apresenta melhor lubricidade e maior número de cetano (Knothe et al., 2008; Barnwal et al.,2005; Garcez et al.,2009).

A forma de produção mais utilizada é por meio da reação de transesterificação de óleos vegetais ou gorduras animais com álcoois de cadeia curta, normalmente metanol ou etanol, na presença de um catalisador básico. Apesar de apresentar boas conversões, na reação de transesterificação ocorre o aparecimento da glicerina como subproduto e impurezas como sais de ácidos graxos, catalisador residual e álcool em excesso (ARDILA et al., 2009). Portanto, estudos de sistemas de extração líquida por solvente para retirada das impurezas se fazem necessários. O conhecimento do equilíbrio de fases entre os componentes envolvidos na 
purificação do biodiesel proporciona informações essenciais para projetar e otimizar os processos de separação.

Nesse trabalho são apresentados dados experimentais de equilíbrio líquido-líquido para o sistema de Biodiesel de Algodão Metílico (BIOAM) + água + metanol, fato justificado pela importância do óleo de algodão para a produção de biodiesel no Brasil, bem como, pela escassez de dados composicionais do referido sistema na literatura.

\section{METODOLOGIA}

\subsection{Materiais}

O biodiesel utilizado no presente trabalho foi produzido no Laboratório de FísicoQuímica Aplicada - Universidade Federal do Ceará, por meio de transesterificação alcalina. A composição do biodiesel foi analisada por cromatografia gasosa com detector de ionização de chama (FID). A água foi destilada e depois deionizada com resistividade igual a 18,2 $\mathrm{m} \Omega$ (deionizador Milipore Simplicity 185, Bedford, EUA) para a retirada de contaminantes orgânicos e inorgânicos. O metanol fornecido pela Synth, com grau de pureza maior que 99,8 $\%$, não foi submetido a processos extras de purificação.

\subsection{Dados de Equilíbrio}

A técnica de quantificação adotada para determinação das composições das fases em equilíbrio foi a densimetria, a qual correlaciona medidas de densidade com as composições, através de uma equação polinomial de segundo grau. Essa metodologia foi validada por Pinheiro et al., 2014. A solubilidade dos sistemas foi testada por meio da curva binodal a qual foi construída através de titulação, partindo de uma mistura inicialmente límpida até a visualização de turbidez indicando a mudança de uma região homogênea para a região de duas fases. A uma mistura binária de composição conhecida foi adicionado um terceiro componente até que o ponto de turbidez se tornasse visível, permanecendo em agitação contínua por 1 minuto. Em seguida, uma amostra foi retirada para análise de densidade. Diferentes pontos da curva de solubilidade foram determinados.

Os componentes da mistura ternária foram pesados diretamente na célula de equilíbrio com o auxílio de uma balança analítica. O volume da cada célula foi completamente preenchido para evitar formação de fase vapor. Os conectores foram vedados com septos de borracha e a célula conectada a um banho termostático que manteve o sistema à temperatura desejada. A mistura foi vigorosamente agitada por três horas para homogeneização completa do sistema. Em seguida, o sistema foi deixado em repouso por quatorze horas, no intuito de garantir que o equilíbrio termodinâmico fosse atingido. No equilíbrio, a formação de duas fases límpidas com interface bem definida foi observada. Uma amostra de $5 \mathrm{~mL}$ foi coletada de cada fase para análise de densidade. Foi utilizado um densímetro da marca Anton Paar, modelo DSA-5000. A fração mássica de cada componente em cada fase de equilíbrio pode ser determinada utilizando as curvas de calibração de densidades obtidas anteriormente.

\subsection{Modelagem Termodinâmica}


Foram utilizados os métodos de contribuição de grupos UNIFAC (Fredenslund, 1975), UNIFAC-Dortmund (Weidlich e Gmehling, 1987) e UNIFAC-LLE (Magnussen, 1981) para a predição dos coeficientes de atividade. No método UNIFAC o coeficiente de atividade é representado por uma soma das contribuições combinatorial e residual. A contribuição combinatorial é devido às diferenças entre a forma e o tamanho das moléculas na mistura; enquanto que a contribuição residual leva em consideração as interações energéticas entre os grupos estruturais que compõem as moléculas da mistura. Os modelos UNIFAC-Dortmund e UNIFAC-LLE são pequenas variações do modelo UNIFAC. A versão UNIFAC-LLE foi desenvolvida por Magnussen (1981), que revisou o modelo UNIFAC, introduzindo diferentes parâmetros de interação de grupo para os sistemas de ELL, mantendo o mesmo conjunto de equações. O modelo UNIFAC-Dortmund apresenta propostas de modificação para o modelo original UNIFAC. A primeira modificação foi no equacionamento do termo combinatorial e a segunda foi a introdução, no termo residual, de parâmetros de interação dependentes da temperatura. Cada componente individual da mistura de biodiesel, ou seja, cada metil éster foi considerado no cálculo de todos os modelos.

O desvio médio quadrático foi encontrado através de uma comparação entre as composições experimentais e calculadas de cada componente em cada uma das duas fases, utilizando a equação dada por:

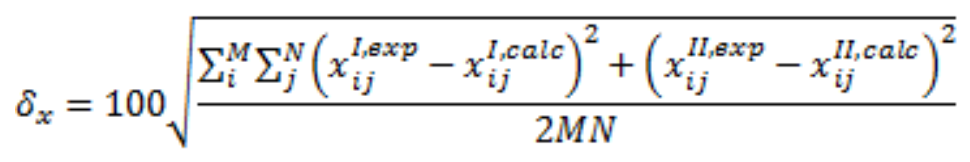

\section{RESULTADOS}

\subsection{Propriedades Físico-Químicas}

Os dados de densidade, índice de acidez e composição do biodiesel metílico de algodão são mostrados na Tabela 1 .

Tabela 1 - Propriedades do biodiesel metílico de algodão

\begin{tabular}{cc}
\hline \multicolumn{2}{c}{ Biodiesel Metílico de Algodão } \\
\hline Densidade a $20^{\circ} \mathrm{C}\left(\mathrm{g} / \mathrm{cm}^{3}\right)$ & 0,8845 \\
Índice de acidez $(\mathrm{mg} \mathrm{KOH} / \mathrm{g})$ & 0,209 \\
Metil éster (\%) & \\
Miristato C14:0 & 0,850 \\
Palmitato C16:0 & 24,08 \\
Esteárico C18:0 & 2,130 \\
Oleato C18:1 & 15,79 \\
Linoleato C18:2 & 56,08 \\
Outros (não identificados) & 1,050 \\
\hline
\end{tabular}




\subsection{Dados de Equilíbrio Líquido-Líquido}

As curvas de calibração foram construídas a partir dos dados de densidade versus fração mássica de cada componente, obtidos nos testes de solubilidade. Os ajustes forneceram parâmetros a, b e c que foram utilizados na quantificação das composições das fases em equilíbrio líquido-líquido. Os valores de densidade e composição da curva de calibração para o sistema biodiesel de algodão, água e metanol são mostrados nas Tabelas 2.

Tabela 2 - Dados de densidade e composição das curvas de calibração para o sistema água (1)

\begin{tabular}{|c|c|c|c|}
\hline$w_{1}$ & $w_{2}$ & $w_{3}$ & Densidade $/\left(\mathrm{g} \cdot \mathrm{cm}^{-3}\right)$ \\
\hline \multicolumn{4}{|c|}{$\begin{array}{c}\text { Água(1) }+ \text { Metanol(2) + BIOAM(3) a } 40^{\circ} \mathrm{C} \\
\text { Fase rica em BIOAM }\end{array}$} \\
\hline 0,0017 & 0,0125 & 0,9859 & 0,865831 \\
\hline 0,0024 & 0,0455 & 0,9521 & 0,862983 \\
\hline 0,0055 & 0,0699 & 0,9245 & 0,861189 \\
\hline 0,0075 & 0,1227 & 0,8698 & 0,856172 \\
\hline 0,0088 & 0,2472 & 0,7440 & 0,844513 \\
\hline 0,0114 & 0,3713 & 0,6173 & 0,832841 \\
\hline 0,0178 & 0,6138 & 0,3684 & 0,809116 \\
\hline 0,0221 & 0,7303 & 0,2476 & 0,800588 \\
\hline 0,0337 & 0,8440 & 0,1223 & 0,792562 \\
\hline \multicolumn{4}{|c|}{ Fase rica em água } \\
\hline 0,9887 & 0,0104 & 0,0009 & 0,990211 \\
\hline 0,9655 & 0,0333 & 0,0012 & 0,986043 \\
\hline 0,8726 & 0,1244 & 0,0031 & 0,970176 \\
\hline 0,7455 & 0,2508 & 0,0036 & 0,948867 \\
\hline 0,6213 & 0,3761 & 0,0027 & 0,926143 \\
\hline 0,5003 & 0,4972 & 0,0025 & 0,901689 \\
\hline 0,3555 & 0,6424 & 0,0022 & 0,868550 \\
\hline 0,2472 & 0,7507 & 0,0021 & 0,841957 \\
\hline
\end{tabular}

As misturas contendo água e biodiesel podem formar emulsões em determinadas concentrações, dificultando agitação e separação das fases. Assim, os pontos de mistura globais do sistema BIOAM + metanol + água foram escolhidos de maneira que a quantidade de água e etanol fosse proporcional à quantidade de biodiesel, ou seja, $50 \%$ de biodiesel. As composições das misturas globais e das fases em equilíbrio são apresentadas na Tabela 3, as linhas de amarração e os dados da curva de solubilidade são representados na Figura 1.

Tabela 3 - Dados de linha de amarração para o sistema água (1) + metanol (2) + BIOAM (3) a $40{ }^{\circ} \mathrm{C}$

\begin{tabular}{|c|c|c|c|c|c|c|c|c|}
\hline \multicolumn{9}{|c|}{ Água(1) + Metanol(2) + BIOAM(3) a $40^{\circ} \mathrm{C}$} \\
\hline \multicolumn{3}{|c|}{ Composição global } & \multicolumn{3}{|c|}{ Fase rica em biodiesel } & \multicolumn{3}{|c|}{ Fase rica em água } \\
\hline $\mathbf{w}_{1}$ & $\mathbf{w}_{\mathbf{2}}$ & $\mathbf{w}_{3}$ & $\mathbf{w}_{1}$ & $\mathbf{w}_{2}$ & $\mathbf{w}_{3}$ & $\mathbf{w}_{1}$ & $\mathbf{w}_{2}$ & $\mathbf{w}_{3}$ \\
\hline 0,3775 & 0,0543 & 0,4456 & 0,0036 & 0,0235 & 0,9729 & 0,8874 & 0,1103 & 0,0023 \\
\hline 0,3110 & 0,0841 & 0,4237 & 0,0037 & 0,0253 & 0,9710 & 0,8297 & 0,1677 & 0,0026 \\
\hline 0,4475 & 0,1271 & 0,3705 & 0,0037 & 0,0287 & 0,9676 & 0,7460 & 0,2511 & 0,0029 \\
\hline 0,4142 & 0,1846 & 0,3104 & 0,0038 & 0,0336 & 0,9626 & 0,6354 & 0,3616 & 0,0030 \\
\hline 0,2531 & 0,2442 & 0,2570 & 0,0038 & 0,0394 & 0,9568 & 0,5238 & 0,4733 & 0,0029 \\
\hline 0,1849 & 0,3088 & 0,1850 & 0,0040 & 0,0496 & 0,9464 & 0,3863 & 0,6112 & 0.0025 \\
\hline
\end{tabular}




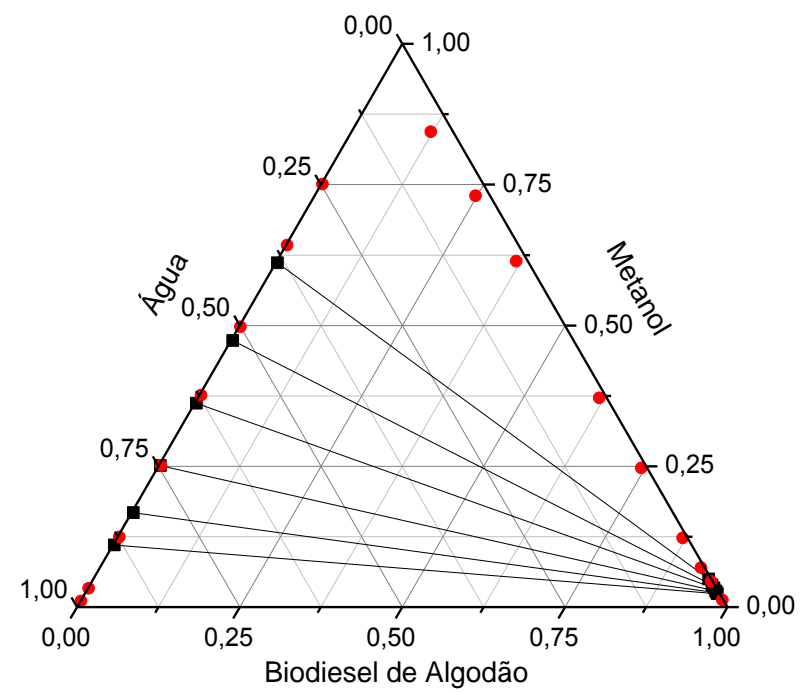

Figura 1 - Linhas de amarração (ロ) e dados de solubilidade (•) do sistema: Biodiesel de Algodão + Água +Metanol a $40^{\circ} \mathrm{C}$.

\subsection{Coeficientes de Distribuição e Seletividade}

Segundo Sandler (1999), o coeficiente de distribuição $(\beta)$ é definido como a razão da concentração de um componente nas duas fases:

$$
\beta=\frac{W(\text { fase } I)}{W(\text { faseII })}
$$

De acordo com Perry e Green (1984), o valor do coeficiente de distribuição é um dos principais parâmetros utilizados para se estabelecer a razão mínima de solvente/alimentação que deve ser manipulada em um processo de extração. A eficiência da extração líquida pode ser avaliada através do coeficiente de separação ou seletividade (S), que pode ser definido como,

$$
S=\frac{\left(x_{B}{ }^{l} / x_{B}{ }^{I I}\right)}{\left(x_{C}{ }^{l} / x_{C}{ }^{H I}\right)}=\frac{\beta_{B}}{\beta_{C}}
$$

na qual, $X_{B}$ é a fração mássica de metanol, $X_{C}$ é a fração mássica de água, A é o soluto (biodiesel metílico de algodão), I representa a fase rica em biodiesel e II representa a fase rica em água; $\beta_{\mathrm{B}}$ é o coeficiente de distribuição do metanol e $\beta_{\mathrm{C}}$ é o coeficiente de distribuição da água. Quando a seletividade é maior que 1, significa que a extração é possível. A Tabela 4 mostra os valores dos coeficientes de distribuição e seletividade para o sistema em estudo.

Os baixos valores dos coeficientes de distribuição obtidos na temperatura analisada indicam alta concentração de álcool na fase rica em água. Esse comportamento pode ser atribuído à alta polaridade e a formação de ligações de hidrogênio entre a água e o metanol, o que torna o metanol mais solúvel em água que no biodiesel. Os valores de seletividade apresentaram-se superiores a 1, isso indica que a água apresenta boa capacidade de extração do metanol presente no biodiesel metílico de algodão. 
Tabela 4 - Coeficientes de distribuição da água $\left(\beta_{1}\right)$, do etanol $\left(\beta_{2}\right)$ e a seletividade $(S)$ para o sistema água (1) + metanol (2) + BIOAM (3), a $40{ }^{\circ} \mathrm{C}$

\begin{tabular}{ccc}
\hline \multicolumn{3}{c}{ Água(1) + Metanol $(\mathbf{2})+\mathbf{B I O A M}(\mathbf{3})$} \\
\hline $\boldsymbol{\beta}_{\mathbf{1}}$ & $\boldsymbol{\beta}_{\mathbf{2}}$ & $\mathbf{S}$ \\
\hline$\left(\mathbf{w}_{\mathbf{1}}{ }^{1} \mathbf{w}_{\mathbf{1}}{ }^{\mathrm{II}}\right)$ & $\left(\mathbf{w}_{\mathbf{2}} / \mathbf{w}_{\mathbf{2}}{ }^{\mathrm{II}}\right)$ & $\left(\boldsymbol{\beta}_{\mathbf{2}} / \boldsymbol{\beta}_{\mathbf{1}}\right)$ \\
\hline 0,0041 & 0,2130 & 52,08 \\
0,0044 & 0,1511 & 34,31 \\
0,0050 & 0,1143 & 23,04 \\
0,0059 & 0,0929 & 15,68 \\
0,0073 & 0,0832 & 11,32 \\
0,0103 & 0,0812 & 7,850 \\
\hline
\end{tabular}

\subsection{Confiabilidade dos Dados Experimentais}

Para verificar a confiabilidade dos dados experimentais de equilíbrio líquido-líquido foi aplicada a correlação proposta por Othmer-Tobias (1942), de acordo com a Equação 4. A confiabilidade dos dados é indicada pela linearidade desta correlação.

- Correlação de Othmer-Tobias:

$$
\ln \left(\frac{1-w_{\mathrm{g}}^{l}}{w_{\mathrm{a}}^{l}}\right)=A+B \ln \left(\frac{1-w_{1}^{I I}}{w_{1}^{I I}}\right)
$$

onde, $w_{3}^{\text {I }}$ representa a fração mássica de biodiesel na fase rica em biodiesel, $w_{1}^{\text {II }}$ representa a fração mássica de água na fase rica em água, A uma constante e B a inclinação da reta. Para que os dados experimentais sejam considerados confiáveis, os valores do coeficiente de correlaçãa $\left(\mathrm{R}^{2}\right)$ devem estar próximos de 1 . Isso pode ser verificado para o sistema biodiesel de algodão, água e metanol a $40{ }^{\circ} \mathrm{C}$, como se pode observar na Tabela 5 onde estão representados os valores de A, B e o coeficiente de correlação $\left(\mathrm{R}^{2}\right)$. Assim os dados podem ser considerados confiáveis.

Tabela 51 - Valores A, B e R² das correlações de Othmer-Tobias para o sistema em estudo

\begin{tabular}{cccc}
\hline & \multicolumn{3}{c}{ Correlação de Othmer-Tobias } \\
\cline { 3 - 4 } Temperatura & A & B & $\mathbf{R}^{2}$ \\
\hline & Biodiesel de algodão + metanol + água \\
\hline $40^{\circ} \mathrm{C}$ & $-3,054$ & 0,280 & 0,97 \\
\hline
\end{tabular}

\subsection{Modelagem Termodinâmica}

Os desvios médios quadráticos globais obtidos com os modelos UNIFAC, UNIFACDortmund e UNIFAC-LLE para o sistema BIOAM + metanol + água a $40{ }^{\circ} \mathrm{C}$ são apresentados na Tabela 6. A presença do grupo $\mathrm{CH}_{3} \mathrm{OH}$ no UNIFAC original faz com que este modelo consiga representar melhor as interações entre os grupos presentes no sistema do que o UNIFAC-LLE, como pode ser observado na Tabela 6. O UNIFAC-Dortmund apresenta parâmetros mais dependentes da temperatura, como o sistema foi estudado em apenas uma temperatura o desvio obtido para este modelo apresentou o maior valor de desvio. 
Tabela 6 - Desvios médios quadráticos globais obtidos com os modelos UNIFAC, UNIFACDortmund e UNIFAC-LLE para o sistema BIOAM + metanol + água a $40{ }^{\circ} \mathrm{C}$

\begin{tabular}{cccc}
\hline & UNIFAC original & UNIFAC-LLE & UNIFAC-Dortmunt \\
\hline Sistema a 40 ${ }^{\circ} \mathbf{C}$ & $\boldsymbol{\Delta w}(\%)$ & $\Delta \mathbf{w}(\%)$ & $\Delta \mathbf{w}(\%)$ \\
\hline BIOAM + Metanol + água & 3,52 & 4,22 & 4,73 \\
\hline
\end{tabular}

A Figura 2 mostra as linhas de amarração experimentais e calculadas para o sistema em estudo. A predição das composições em equilíbrio apresentou melhor representatividade para a fase com maior concentração de biodiesel, isso ocorre devido à grande concentração de metanol na fase rica em água o que aumenta a formação de ligações de hidrogênio tornando a fase não ideal.

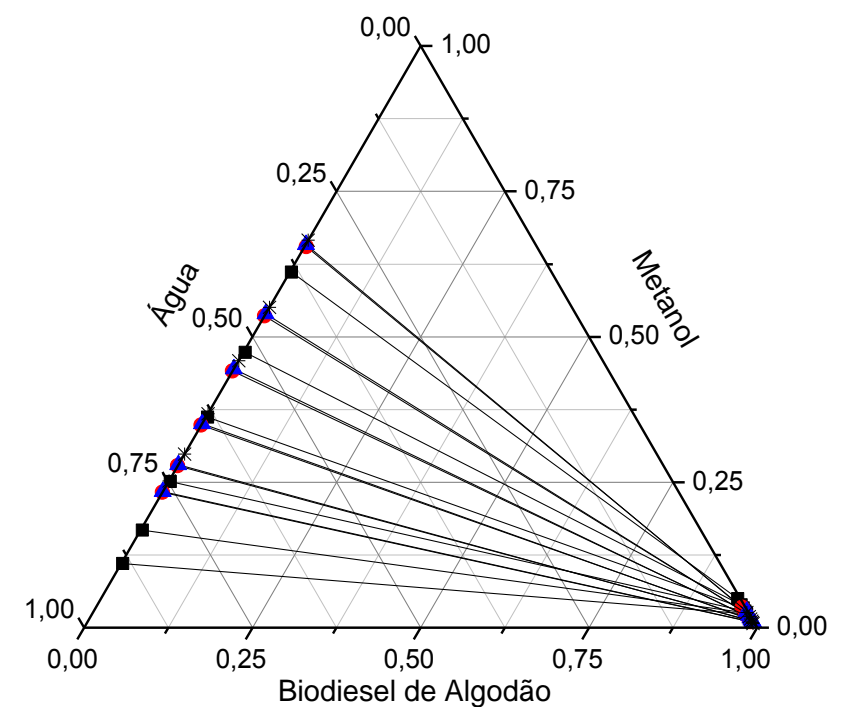

Figura 2 - Linhas de amarração experimentais e calculadas pelos modelos termodinâmicos. (घ) UNIFAC Original; (•) UNIFAC-Dortmund; ( $\boldsymbol{\Delta}$ ) UNIFAC-LLE e (*) experimental.

\section{CONCLUSÃO}

Dados de equilíbrio líquido-líquido para o sistema biodiesel de algodão, metanol e água a $40{ }^{\circ} \mathrm{C}$ foram obtidos por meio da densimetria. A confiabilidade dos dados experimentais foi comprovada através da correlação de Othmer-Tobias que apresentou coeficientes de regressão próximos da unidade. Os coeficientes de distribuição para todos os sistemas analisados apresentaram baixos valores indicando a distribuição preferencial do metanol nas fase rica em água. Os valores de seletividade obtidos para o sistema estudado foram superiores a $1 \mathrm{em}$ todas as composições testadas, portanto, pode-se concluir que a água apresenta boa capacidade de extração do metanol. Os modelos termodinâmicos de contribuição de grupos UNIFAC, UNIFAC-Dortmund e UNIFAC-LLE apresentaram baixos valores de desvios médios: 3,52, 4,22 e 4,73, respectivamente. De acordo com os desvios calculados o modelo UNIFAC possui melhor representatividade para o sistema estudado. 


\section{REFERÊNCIAS BIBLIOGRÁFICAS}

ARDILA, Y, C. Sistemas de extração líquido-líquido para processos de purificação de biodiesel. 2009. Dissertação (Mestrado em Engenharia Química) - Universidade Estadual de Campinas, Campinas, 2009.

BARNWAL, B. K.; SHARMA, M. P. Prospects of biodiesel production from vegetable oils in India, Renewable and Sustainable Energy Reviews, v. 9, p. 363-378, 2005.

FREDENSLUND, A.; JONES, R. L.; PRAUSNITZ J. M. Group-contribution estimation of activity coefficients in nonideal liquid mixtures. AIChE J, v. 21, p.1086-99, 1975.

GARCEZ, C. G.; VIANNA, J. S. Brazilian Biodiesel Policy: Social and Enviromental considerations of sustainability, Energy, v. 34, p. 645-654, 2009.

KNOTHE, G.; Van GERPEN, J. H.; Manual do Biodiesel. 2. ed., AOCS Publicações, Urbana, 2008.

MAGNUSSEN, T. UNIFAC parameter table for prediction of liquid-liquid equilibria. Ind Eng Chem Process Des Dev, v. 20, p. 331-9, 1981.

OTHMER, D. F.; TOBIAS, P. E. Tie-line correlation. Ind. Eng. Chem. Re. v. 34, p. 693-696. 1942.

PERR, /Y. R. H.;/GREEN, D. P. Chemical Engineering Handbook, 6, ed, 1984.

PINHEIRO, R. S; BESSA, A. M./M., QUEIROZ, B. A; DUARTE, A. M. S. F; Sant'Ana, /H. B; SANTIAGO-AGUIAR, R./S. Optimization of the methylic biodiesel purification process by intermediate of liquid-liquid equilibrium data for ternary systems containing methanol + water + (soybean, corn or brown shell of coconut) biodiesel. Fluid phase Equilib., v. 361, p. 30-36, 2014.

SANDLER, S. I. Models for Thermodynamics and Phase Equilibria Calculations, New York: M, Dekker, 1999.

WEIDLICH, U.; GMEHLING, J. A modified UNIFAC model. 1.Prediction of VLE, hE, and y m. Ind Eng Chem Res, v. 26, p. 1372-81, 1987. 